\title{
Mixed messages: an evaluation of NHS Trust Social Media Policies in the North West of England
}

\section{Introduction}

The NHS information strategy promotes the use of Social Media (SoMe) to engage online with patients and clients in innovative ways (Department of Health, 2012) The Word of Mouth Mammogram e Network (WoMMeN) breast screening SoMe hub was developed in response to this strategy (Hill et al., 2016).

Funded by the College of Radiographers Industry Partnership Scheme (CoRIPS), the WoMMeN team has been working closely with mammography practitioners, and other NHS Breast Screening employees who communicate with clients about mammography, to explore their attitudes to engaging with the breast screening client population online. A key finding from these exploratory workshops has been that some practitioners have felt actively discouraged from using SoMe by their Trust communication policies.

This is at odds with the National Health Service (NHS) strategy and the Society and College of Radiographers' SoMe Guidelines (Department of Health, 2012; Society and College of Radiographers, 2015), and also with population behaviour, as SoMe use is ubiquitous; with 30 million UK Facebook users reported in 2014 (www.statista.com) and 305 million worldwide Twitter users in 2015. Fifty per cent of UK Internet users report looking for health related information as a daily activity (Office of National Statistics, 2016) and the NHS, as an overall health service provider is looking to meet that demand, as well as harnessing the fast, flexible and direct nature of SoMe to engage with stakeholders collaboratively (White, 2012).

The CoRIPS findings will be reported in full elsewhere, but this employers barrier to SoMe use alleged by the breast screening community, despite evidence of increased demand for information and engagement by clients and other stakeholders (Robinson et al., 2016) warranted further consideration.

\section{Aim}

The aim of the study was firstly to investigate whether the barrier to SoMe use by employer policies was genuine, or a misperception by employees. This required interrogating the policy content to uncover any implicit or explicit instructions to staff about SoMe use.

Secondly, from this interrogation, the study intended to generate a theory to increase understanding of the reported anxieties and behaviour through a comparative analysis of the reported source of the anxiety: the textual data of the policies themselves.

Thirdly, the study aimed to illuminate whether local employer policy effectively aligned with national strategy in the respect of using SoMe for engaging with stakeholders.

\section{Methodological Approach}

In the absence of a definite hypothesis but with an aim of increasing understanding of the situation by generating a theory; the objectives led the researchers towards a qualitative rather than a quantitative approach to the study. 
The researchers considered their own objectivity and the potential for bias within the study when choosing a methodology. The researchers, as breast screening practitioners, are an intrinsic part of the study population; and are familiar with working within the NHS policies investigated. However, they had no a priori knowledge of individual employers' SoMe policies, and unlike the workshop participants, they did not feel that using SoMe put them at odds with their employers.

This scenario was felt to lend itself to a form of grounded theory approach to policy analysis: as stated by Glaser (1978), the purpose of grounded theory is "to account for a pattern of behaviour which is relevant and problematic for those involved" (Glaser, 1978) p.93). Grounded theory was developed in 1967, and generates theories to explain a phenomenon (Glaser \& Strauss, 1967). Since derived, the theory has undergone refinements and deviations from the classic theory by several authors; but essentially it is a deductive-inductive theoretical framework, using continuous, detailrich data collection and comparative analysis to code and categorise the data, so that theories can emerge about the studied phenomenon (Charmaz, 2006).

The main points of difference occur with the inclusion of prior knowledge to the literature; classically, the researcher would not allow this to inform the research, but later versions of the methodology permit flexibility on this point (Stern, 1980). It is important to acknowledge that flexibility and an open mind are assets when using grounded theory.

The researchers considered their relationship with the study topic with regard to the three 'positions' as defined by (Reed \& Procter, 1995), an 'outsider', 'hybrid' or 'insider', with each position directly relating to the amount of professional knowledge acquired previously. The researchers classed themselves as 'insiders', with knowledge of the wider professional literature as well as the use of SoMe as breast screening practitioners.

Positioned thus, the researchers decided to preclude a targeted literature review prior to data collection, as within grounded theory the literature is also treated as data, and could direct the study and bias the emerging theory from the policy data analysis (McGhee, Marland, \& Atkinson, 2007)

\section{Ethical Considerations}

Informed consent had been obtained from the participants at the CoRIPS workshops, who understood that they were also to be research subjects. Policies were publically available online or obtained from the communications team, with permission to audit. Ethical approval was not deemed necessary for this, as it was framed as service evaluation.

\section{Method}

As 'insiders', the researchers confined the setting to the ten NHS Trusts providing breast screening in their own North West region of England, and nine policies on internet and SoMe use were acquired. The tenth Trust did not have a policy.

The unit of analysis, the policy content, was considered to be the raw data, and an initial familiarisation resulted in the researchers meeting and agreeing that the overall tone of the policy content was either discouraging, encouraging or enabling. A simple framework was thus derived from the data and a matrix created on an Excel spreadsheet. The researchers undertook the tonal analysis separately before meeting to compare their respective results. The matrices were identical in $85 \%$ of cells, and the researchers discussed their differences and similarities in approach before the next stage. Framework analysis was developed for social policy research, and allows analysis of qualitative data in a quantifiable manner, easing analysis of differences and similarities (Gale, Heath, Cameron, Rashid, \& Redwood, 2013). 
The policy content was then re-examined in detail and coding was undertaken; 19 different codes were identified and agreed upon by both researchers. Content analysis was aided by Computer Aided Qualitative Data Analysis (CAQDAS); in this case nVivo software (QSR International, n.d.).

\section{Results}

\section{Tonal Analysis}

Overall, Trust Policies were varied in content but in tone were mostly discouraging by being prohibitive; one (source 2 ) was contradictory, with prohibitive and encouraging tones, as well as enabling staff by offering training (Figure 1 ).

Insert Figure 1: Overall Tone of Policy matrix

\section{Prohibitive statements:}

'...should not discuss work-related matters...' Source 1

'Inappropriate use of social media is considered misconduct....' Source 7

\section{Encouraging statements:}

'The intention of this policy is not to stop or dissuade Trust staff from using social media personally or professionally...' Source 2

'...may use designated facilities provided by the trust for their private use of social media...' Source 2

\section{Enabling statements:}

'Workshops may be arranged for staff who find it helpful..... Source 2

\section{Detailed Content Analysis}

The policies addressed nineteen separate issues in total, the most frequently occurring was 'upholding the reputation of the Trust' (Figure 2). Surprisingly to the researchers, although mentioned often, patient confidentiality did not appear to be the most concerning issue within any of the Policies reviewed. Professional conduct and behaviour was addressed; but conduct rules were extended in some cases to personal life too. Only one Trust policy mentioned training staff in the use of SoMe.

Insert Figure 2: Frequency of Issues mentioned

The data also revealed how comprehensively the use of SoMe was addressed. All the policies addressed the issue of upholding the Trust's reputation. Information Governance, illegal acts and confidentiality were also covered by all policies. Upholding staff personal reputation was considered by only four Trusts. Allowing access to SoMe or the internet was only addressed by five of the Trusts. However, monitoring internet and SoMe traffic was enforced by eight policies. In contrast, only one employer considered offering training on how to use SoMe.

Insert Figure 3: Number of Sources Addressing Issues

\section{Literature Review}

It was noted that a few themes were emerging from the data, and it was determined that, in keeping with standard practice for Grounded Theory research, at this point the literature should be consulted so that the themes could be refined and reviewed. 
Searches were undertaken on MEDLINE and Google Scholar using terms 'social media' AND 'policy' AND 'healthcare'. Results indicated that very few papers had been published about SoMe policies within healthcare, and those were from the USA. The healthcare system and culture are very different in the UK from that of the US; and thus we propose that the subject matter may well be treated very differently.

In a 2010 quantitative study (Kind, Genrich, Sodhi, \& Chretien, 2010), 10\% of US medical schools were found to have policies addressing the use of SoMe by students (sample size $n=132$ ), however, as in our study, it was shown that the policies varied in their comprehensiveness and their tone ranging from prohibitive to encouraging - and $38 \%$ of the policies studied defined how online behaviour should be by using deterrence and prohibition. The remaining policies were more reflective in tone, guiding students by asking them how they would like to be perceived by others online. The study has limitations in the small sample size and descriptive nature; policy content was not analysed in detail to discover the underlying themes and reasoning behind the policies (Kind et al., 2010).

Another mixed methods study surveyed the heads of US dental schools about their school's and students' use of SoMe. This survey discovered that $50 \%$ of responding schools had a SoMe policy, and that violations of policy had occurred; mostly because of personal conduct issues. Although again the low response rate precluded generalisation to the whole of the US, five SoMe policies were obtained and themes were extracted. These were:

- Privacy

- Intellectual property

- Use of branding / misrepresentation

- Professional standards

- Authorising official school Social Media

- Disciplinary Action

- Legal violations

- Behavioural guidelines

The study did not, however, analyse what weighting the policies leant to these themes; but did find that privacy, use of branding / misrepresentation was common to all policies, suggesting that comprehensiveness and tone were again variable (Henry \& Webb, 2014). The themes found do mirror what we found, but not all of our themes were duplicated in that study.

A similar study to our own was discovered whereby the SoMe policies of European Public Health organisations were coded and analysed. Twenty-one external SoMe policies were analysed, the themes found were:

- General Information

- Policy Scope

- Regulations

- Legal Aspects

- Public Health

However, the study found that the most prevalent constituents of the policies were disclaimer, copyright and privacy; but did not declare which themes these belonged to. The themes from our study did not seem to easily fit the reported themes (Fast, Sørensen, Brand, \& Suggs, 2015). 
A commentary was found that did elicit four themes that were purported to be of concern to healthcare organisations, and here we start to see some parallels with our study data. The four main themes were:

- Reputation Issues

- Privacy Issues

- Productivity Issues

- Training \& Education Issues

(Cain, 2011)

As this is a commentary, it is not possible to examine the evidence and analyse whether it is based on policies or merely opinion. However, the researchers considered it to be of value as the categories are broad ones, and our own themes could fit with ease into them.

At this point, after analysing what little data the literature review had added overall, the researchers compared it with the study data and attempted to refine a theory.

Results:

Using a Mindmap to ease visualisation (Figure 4) (http://mindmapmaker.org), the framework was therefore re-reviewed and the themes condensed further into broad categories, four of which were identical to those suggested by (Cain, 2011). The data that was unaccounted for by Cain's commentary condensed into another category: Conduct \& Behaviour, as suggested by a US study (Henry \& Webb, 2014).

The compressing of our data into the final five themes was an iterative process, which evolved from comparing our data with that suggested by the literature; no exact fit was found with any other study, but we suggest that our data supports that these issues are the ones that the study policies address.

Insert Figure 4: MindMap

Figure 5 illustrates the condensed framework, with re-categorised codes from Figure 2 in the five umbrella categories. It can be observed that Reputation, Security and Conduct \& Behaviour are the most addressed categories within the sample, with Training \& Education remaining overlooked. The data supports that the SoMe policies appear to overly restrict and control behaviour, with an emphasis on security and protecting the reputation of the organisation. The hesitancy expressed by radiographers about using SoMe can be partly attributed to their employers' policies.

Insert Figure 5: Condensed Framework.

The emergent theory from the data demonstrates that policies address five categories overall, but do not address them equally. Training \& Education is neglected in comparison to Security concerns, and that this could explain the findings from the CoRIPS workshops.

\section{Discussion:}

The overall purpose of an organisational policy is to ensure best practice is followed in a consistent manner, with risks to the organisation kept to a minimum. It is a method of formal communication that informs employees of rules and regulations (Husin \& Hanisch, 2011). Thus, policies are used as a method of control, containment and perceived risk reduction. 
This could be seen to be at odds with SoMe; which is based upon sharing content, opinions and connections freely and widely by users, with a minimum of control and structure; creating a tension between that and organisational policy goals (Husin \& Hanisch, 2011). Health professionals are also regulated by their professional bodies, and these have also created SoMe guidelines, which may not align with their employer's policies. For instance, the Society \& College of Radiographers' guidelines are encouraging, whereas our results indicate that a radiographer's employer's policies are not, creating another source of tension (Society and College of Radiographers, 2015).

It can be seen that our data supports the assertion by the breast screening community that practitioners within breast screening services are discouraged from using SoMe by overly prohibitive policies, aiming to tightly control the flow of information from the organisation. The picture overall is one of mixed messages, with practitioners under conflicting rules and regulations; however, working within the daily confines of their employer's policies perhaps gives these more influence over behaviour modification.

Within the Radiography profession, research in the field of SoMe has been rare - an attempt at a systematic review failed due to zero identifiable papers. Extending the search to allied healthcare found that SoMe was being used as a pedagogical tool and for professional development; although it cannot be said definitively that radiographers are included here because of the lack of evidence (Lawson \& Cowling, 2015). Despite this evidential lack, a successful online radiography journal club does exist for research collaboration and Continuing Professional Development (CPD) purposes without geographical barriers (Nightingale, 2016). The \#WeCommunities on Twitter also undertake various 'Tweetchats' for similar purposes (http://www.wecommunities.org), although there is no evidence to link improved patient outcomes with this type of CPD as yet.

The allied healthcare regulatory body, the Health \& Care Professions Council (HCPC) requires mandatory CPD (Health \& Care Professions Council, 2016). An organisation employs the radiographer with the pre-requisite of HCPC registration. It is therefore unhelpful to limit access to a pedagogical and collaborative resource that may well result in worldwide recognition for that organisation if associated with research by one of their employees. The literature review as part of this study discovered that further research is required to build the evidence base and convince employers that patient and employee outcomes can be sufficiently enhanced by the use of SoMe.

Earlier work by (Robinson et al., 2016) established that health service users wanted to engage with health professionals online, specifically in the area of breast screening. Professionals work under policies that generally discourage this, as our data demonstrated that healthcare organisations prioritise protecting their reputation and information security. Six domains of professionalism have been defined, and they include such tenets as responsibility, patient relations, collaboration, and teamwork (Rogers \& Ballantyne, 2010); nowhere is the caveat 'but not online' included within notions of professionalism. It is arguable that those most well-equipped to answer health queries are not the Trust Communications team but the professionals who hold the specialised knowledge; with training and education in online networking for those unsure of how certain platforms work, we put the case forward for professionals to take a more active role in engaging with patients, and that more employer policies should address this issue than currently do.

The notion of professionalism online is also under scrutiny as the case for e-professionalism is advanced. Conduct rules and regulations, although helpful, should not be overly prescriptive as it is suggested that a more reflective approach will be more successful in developing a critical viewpoint to posting online content and acquiescence to the policy (Fenwick, 2014) (Cain, 2011). However, within this study, a curious catch-all phrase was found in a few separate policies, that of 'work- 
related matters', accompanied with exhortations not to discuss these online. This deliberately vague phrase adds little to practitioner's comprehension when deciding whether to use SoMe or not.

The policies examined were found to be solely authored; to make a policy more effective it is suggested that all stakeholders be involved, and the process of policy development transparent (Junco, 2011) (Cain, 2011). This may well address the findings of skewed focus in this study, whereby the organisations belied their priority with the frequency with which they exhorted protection of the organisational reputation, as opposed to training and education. This skewed focus and lack of stakeholder engagement at policy formation stage may explain why local policies do not effectively align with national policy; the study demonstrated that most employers preferred to address the flow of information to protect their reputation and brand rather than the issue of engaging with clients online.

\section{Conclusion:}

The emergent theory from the data demonstrated that policies addressed five broad categories of issues, and that these are reasonable, and according to current literature should be expected to be included in a policy in order to effectively perform the overall function of an organisational policy. However, the categories are not given equal weighting within the policies, leading to a skewed focus on overly prescriptive and prohibitive policies which stymie the use of SoMe by staff. This in turn, leads to a lack of engagement with stakeholders, which national strategy is attempting to encourage.

Based on our analysis, practitioners are working under three differing bodies, their employer, their professional body and the NHS as an organisation; each of these entities appears to have stamped their own opinion onto rules and regulations as to how staff approach and utilise SoMe; and each of these policies differ in focus. This is understandably leading to confusion, with practitioners receiving mixed messages about how to use this ubiquitous method of communication.

It was further shown that policies varied tremendously across one small region in content and comprehensiveness, with a skew in focus toward security and protecting reputation of each Trust. It is assumed here, that variation is the case across the UK, adding further to the confusion.

\section{Limitations:}

There were several study limitations. Firstly related to sample bias; a convenience sample is a nonprobability sample, which is inherently biased (Bryant \& Charmaz, 2010).

Secondly related to researcher bias; both researchers who performed the analysis were part of the WoMMeN team, a group that is encouraging online communication between mammography practitioners and breast screening clients. Prior to the study starting these researchers had experienced the barriers that practitioners faced and worked within the geographical area under study. However, within the constraints of grounded theory, bias was kept to a minimum and theoretical sensitivity was maintained, the researchers practiced reflexivity by remaining openminded about the findings, and acknowledging that their own experience might colour the results. Researchers coded separately and met up to check that their understanding of the emergent theory was in agreement, in an attempt to reduce bias.

Thirdly, data saturation was not able to be adequately measured due to the nature of the sampling. This may reduce rigour of the study.

\section{Recommendations}


Following the results of this Policy review several recommendations can be made; firstly, the National Information Board should be consulted in order to base the SoMe policies according to current health information requirements, as currently policies do not align with the NHS vision of using SoMe to communicate with stakeholders.

It would appear that health practitioners in addition to patient groups should be consulted in order to revise and update policies in line with current digital health trends. Robust independent assessment of policies should also be undertaken to ensure quality, safety and effectiveness of the updated policies. Finally, SoMe training should be included as mandatory training for all members of staff.

The study has demonstrated the need for further research in both the effectiveness of SoMe in influencing client outcomes, and also a need for further research into the apparent lack of enthusiasm within the radiography profession for using it; it has to be acknowledged that factors other than employer policies are influencing behaviour of this particular group within healthcare.

\section{References}

Bryant, A., \& Charmaz, K. (2010). The SAGE Handbook of Grounded Theory: Paperback Edition. SAGE Publications. Retrieved from https://books.google.co.uk/books?id=OrgZjp9CoN8C

Cain, J. (2011). Social media in health care: the case for organizational policy and employee education. American Journal of Health-System Pharmacy, 68(11), 1036.

Charmaz, K. (2006). Constructing Grounded Theory: A Practical Guide through Qualitative Analysis (Introducing Qualitative Methods series). Sage Publications Ltd. Retrieved from citeulike-articleid:2806115

Department of Health. (2012). The power of information: Putting all of us in control of the health information we need. London: Department of Health. Retrieved from https://www.gov.uk/government/uploads/system/uploads/attachment_data/file/213689/dh_13420 5.pdf

Fast, I., Sørensen, K., Brand, H., \& Suggs, L. S. (2015). Social Media for Public Health: An Exploratory Policy Analysis. The European Journal of Public Health, 25(1), 162-166. Retrieved from http://eurpub.oxfordjournals.org/content/25/1/162.abstract

Fenwick, T. (2014). Social media and medical professionalism: rethinking the debate and the way forward. Academic Medicine : Journal of the Association of American Medical Colleges, 89(10), 1331-1334. http://doi.org/10.1097/ACM.0000000000000436

Gale, N. K., Heath, G., Cameron, E., Rashid, S., \& Redwood, S. (2013). Using the framework method for the analysis of qualitative data in multi-disciplinary health research. BMC Medical Research Methodology, 13, 117. http://doi.org/10.1186/1471-2288-13-117

Glaser, B. G. (1978). Theoretical sensitivity: advances in the methodology of grounded theory. Sociology Press. Retrieved from https://books.google.co.uk/books?id=73-2AAAAIAAJ

Glaser, B. G., \& Strauss, A. L. (1967). The Discovery of Grounded Theory: Strategies for Qualitative Research. Aldine Publishing Company. Retrieved from https://books.google.co.uk/books?id=oUxEAQAAIAAJ 
Health \& Care Professions Council. Standards of conduct, performance and ethics (2016). United Kingdom. Retrieved from http://www.hcpc-

uk.org/assets/documents/10004EDFStandardsofconduct,performanceandethics.pdf

Henry, R. K., \& Webb, C. (2014). A survey of social media policies in U.S. dental schools. Journal of Dental Education, 78(6), 850-5. Retrieved from http://www.ncbi.nlm.nih.gov/pubmed/24882770

Hill, C., Robinson, L., Griffiths, M., Mercer, C., Scragg, B., Shires, G., ... Wray, J. (2016). A mammographer-led online information service for breast clients: exploring the professional's perspective. In Symposium Mammographicum Liverpool UK. Poster presentation.

Husin, M. H., \& Hanisch, J. (2011). Social media and organisation policy (someop): finding the perfect balance. In V. K. Tuunainen, M. Rossi, \& J. Nandhakumar (Eds.), ECIS. Retrieved from http://dblp.unitrier.de/db/conf/ecis/ecis2011.html\#HusinH11

Junco, R. (2011). The need for student social media policies. Retrieved from http://er.educause.edu/articles/2011/2/the-need-for-student-social-media-policies

Kind, T., Genrich, G., Sodhi, A., \& Chretien, K. C. (2010). Social media policies at US medical schools. Medical Education Online, 15. http://doi.org/10.3402/meo.v15i0.5324

Lawson, C., \& Cowling, C. (2015). Social media: The next frontier for professional development in radiography. Radiography, 21(2), e74-e80.

http://doi.org/http://dx.doi.org/10.1016/j.radi.2014.11.006

McGhee, G., Marland, G. R., \& Atkinson, J. (2007). Grounded theory research: literature reviewing and reflexivity. Journal of Advanced Nursing, 60(3), 334-342. http://doi.org/10.1111/j.1365-

2648.2007.04436.x

Nightingale, J. (2016). Radiography research as a global community. Radiography, 21(2), 108-109. http://doi.org/10.1016/j.radi.2015.03.006

Office of National Statistics. (2016). Internet access - households and individuals: 2016. Retrieved September 1, 2016, from

https://www.ons.gov.uk/peoplepopulationandcommunity/householdcharacteristics/homeinterneta ndsocialmediausage/bulletins/internetaccesshouseholdsandindividuals/2016\#activities-completedon-the-internet

QSR International. (n.d.). nVivo Software product. Retrieved May 1, 2016, from http://www.qsinternational.com/nvivo-product

Reed, J., \& Procter, S. (1995). Practitioner research in health care. http://doi.org/10.1007/978-14899-6627-8_1

Robinson, L., Griffiths, M., Wray, J., Ure, C., Shires, G., Stein-Hodgins, J. R., ... Hilton, B. (2016). Preparing women for breast screening mammography: A feasibility study to determine the potential value of an on-line social network and information hub. Radiography, 21(4), 308-314.

http://doi.org/10.1016/j.radi.2015.07.004

Rogers, W., \& Ballantyne, A. (2010). Towards a practical definition of professional behaviour. Journal of Medical Ethics , 36(4), 250-254. http://doi.org/10.1136/jme.2009.035121 
Society and College of Radiographers. (2015). SoMeRAD: Guidance for the radiography workforce on the professional use of Social Media. London: Society \& College of Radiographers.

Stern, P. N. (1980). Grounded Theory Methodology: Its Uses and Processes. Image, 12(1), 20-23. http://doi.org/10.1111/j.1547-5069.1980.tb01455.x

White, C. (2012). Using Social Media to engage, listen and learn. London: NHS Networks. Retrieved from https://www.networks.nhs.uk/nhs-networks/smart-guides/documents/Using social media to engage- listen and learn.pdf 